\title{
Auvergnats malgré eux. Prisonniers de guerre et déserteurs étrangers dans le Puy-de-Dôme pendant la Révolution française (1794-1796)
}

Annie Crépin

\section{(2) OpenEdition \\ 1 Journals}

Édition électronique

URL : https://journals.openedition.org/ahrf/1066

DOI : 10.4000/ahrf.1066

ISSN : 1952-403X

Éditeur :

Armand Colin, Société des études robespierristes

\section{Édition imprimée}

Date de publication : 1 mars 2001

Pagination : 138-140

ISSN : 0003-4436

\section{Référence électronique}

Annie Crépin, « Auvergnats malgré eux. Prisonniers de guerre et déserteurs étrangers dans le Puy-deDôme pendant la Révolution française (1794-1796) », Annales historiques de la Révolution française [En ligne], 323 | janvier-mars 2001, mis en ligne le 10 avril 2006, consulté le 22 avril 2022. URL : http:// journals.openedition.org/ahrf/1066; DOI : https://doi.org/10.4000/ahrf.1066

Ce document a été généré automatiquement le 22 avril 2022

Tous droits réservés 


\title{
Auvergnats malgré eux. Prisonniers de guerre et déserteurs étrangers dans le Puy-de-Dôme pendant la Révolution française (1794-1796)
}

\author{
Annie Crépin
}

\section{RÉFÉRENCE}

Frédéric Jarrousse, Auvergnats malgré eux. Prisonniers de guerre et déserteurs étrangers dans le Puy-de-Dôme pendant la Révolution française (1794-1796), publications de l'Institut d'Études du Massif Central, collection Prestige, fascicule n XIII (1999) XII-249p.

1 Cet ouvrage est issu d'un mémoire de maîtrise réalisé sous la direction de Ph.Bourdin, à l'Université de Clermont II. Ph. Bourdin, qui le préface, rappelle que cette étude s'inscrit dans un contexte national qui voit la Révolution, d'abord universaliste, basculer très vite après l'entrée en guerre dans une attitude de suspicion à l'égard de l'étranger au point que ce terme désigne tout à la fois le non-national, le citoyen déchu, le traître à la Patrie. Il rappelle aussi les perspectives dans lesquelles s'est placé $\mathrm{F}$. Jarrousse qui a eu le mérite de prospecter un champ historiographique peu fréquenté jusque-là, quoiqu'investi récemment par les historiens américains, et d'en proposer une approche renouvelée par les récentes analyses sur le patriotisme et le nationalisme. L'auteur a tenté, dans le cadre d'un département, une esquisse des acculturations entraînées par les guerres de la Révolution, le pluriel montrant qu'il s'agit le plus souvent de résistances.

2 En introduction, l'auteur précise son projet et les sources sur lesquelles il s'est appuyé pour comprendre le devenir des 2000 prisonniers étrangers qui ont vécu dans le Puyde-Dôme. Il souligne que l'historiographie s'est d'abord intéressée aux Français captifs à l'étranger, que les étrangers retenus en France n'ont donné lieu qu'à de brefs articles 
et qu'on ne dispose toujours pas d'une synthèse sur la captivité militaire. Le Puy-deDôme fut même «victime d'une amnésie paradoxale puisque, $d u$ XvIII ${ }^{\mathrm{e}}$ siècle à la Seconde Guerre mondiale, il accueillit des prisonniers de guerre. Un érudit local, Marcheix, pionnier oublié, consacra cependant en 1914-1915 un article à cette question mais celui-ci reposait surtout sur des sources officielles. Sans les négliger, F. Jarrousse a aussi utilisé des listes nominatives qui permettent d'appréhender les individus et leur histoire personnelle voire les composantes sociologiques et anthropologiques de celles-ci. Cette étude qui s'élargit aussi aux déserteurs étrangers concerne la période de 1794 à 1796 pour laquelle les sources sont le plus abondantes.

3 Trois parties structurent l'ouvrage. La première est consacrée à l'histoire personnelle et collective des prisonniers et des déserteurs. Le premier chapitre étudie la provenance par fronts des différents contingents de captifs et montre que le département fut inégalement affecté par leur présence. Zone de «détention idéale, car relativement éloigné des frontières et surtout des lieux de combat, le département n'a pas de dépôts dans touts ses districts certains, tel Riom, accueillent beaucoup d'étrangers, d'autres, celui de Montaigut, peu, d'autres enfin, celui de Besse, pas du tout. La captivité des militaires étrangers est un phénomène qui touche surtout la plaine de la Limagne plus peuplée, son importance décroît quand on s'éloigne de Riom et de Clermont. Les contacts avec la population française n'ont donc pas eu lieu partout.

4 Dans les deux chapitres suivants, l'auteur tire le meilleur parti des sources nominatives, en dépit de leurs lacunes et de leurs déformations. Il étudie d'abord le destin de ces «Autrichiens et Hollandais puis des prisonniers de plus en plus nombreux des armées des Alpes et des Pyrénées-Orientales, confirmant au passage ce que l'on sait des armées d'Ancien Régime mode de recrutement, nombre anormalement élevé d'orphelins de mère, présence de non-combattants voire de femmes et d'enfants, cosmopolitisme, plus marqué cependant pour l'armée des Habsbourg que pour celle du roi du Piémont. L'historien note toutefois que le pays d'origine du prisonnier est souvent confondu avec la puissance qu'il sert. Le troisième chapitre se fonde sur des méthodes historiographiques renouvelées puisqu'il est une approche anthropologique et, mais pour les déserteurs seulement, sociologique, vu l'état des sources. De nombreux tableaux qui ne concernent pas seulement ce chapitre, puisque le livre en comporte trente-sept au total, synthétisent les résultats auxquels est parvenu $\mathrm{F}$. Jarrousse.

5 La deuxième partie aborde les conditions d'existence de ces captifs. Elle est également divisée en trois chapitres, le premier s'intéressant à «ceux de l'autre côté, c'est-à-dire les surveillants des dépôts. L'auteur insiste sur la faiblesse du système et même ses contradictions, la Convention ayant prévu en 1793 de l'organiser autour de sousofficiers, le Comité de salut public leur ayant préféré en 1794 des hommes de la garde citoyenne. L'organisation est également obérée par le manque de gardiens dont la tâche est, au demeurant, délicate car le travail de surveillance s'accompagne d'une dévorante activité comptable et épistolaire. Certains, dont l'historien brosse de vivants portraits, s'accommodent cependant de ce système et y font carrière.

6 Le chapitre suivant -un des plus riches du livre- est consacré au travail des prisonniers. Tout le monde trouve son compte à l'emploi de prisonniers la République d'abord, car cette main-d'œuvre utilement canalisée et détournée d'occasions de se rebeller, sert l'économie d'un pays où la guerre est mangeuse d'hommes, les employeurs ensuite -au 
premier chef les agriculteurs- car, si les occupations sont diverses, c'est l'agriculture qui retient le plus de captifs. Pour les déserteurs, en outre, qui ne reçoivent aucun secours, le travail est une nécessité absolue. Plus encore que le tableau des différents secteurs d'activité, c'est la peinture des liens qui se nouent entre étrangers et habitants du Puy-de-Dôme qui est passionnante. Mais l'auteur relativise la place des étrangers au cœur d'une Révolution qui est culturelle, en remarquant que ces contacts sont sporadiques. Le dernier chapitre évoque de façon vivante la condition de ces captifs, en passant en revue tous les aspects de leur vie matérielle logement, alimentation, habillement et solde. F. Jarrousse conclut à l'extrême précarité de leur vie quotidienne, nullement due, pourtant, à une quelconque malveillance de la part des autorités.

7 Après avoir dépeint le présent des prisonniers, l'historien s'attache dans une troisième partie à évoquer leur devenir, qui a pu les conduire à la mort. Le premier chapitre de cette partie met en effet en lumière le mauvais état de santé de ces hommes, mal vêtus et mal nourris, vivant dans de mauvaises conditions d'hygiène les tableaux qui illustrent ce chapitre sont précieux pour le lecteur. Tous ne meurent pas et certains, cherchant à échapper à leur condition, s'évadent. C'est le sujet du chapitre suivant qui montre ainsi que quelques captifs ont un comportement proche du billardage des armées d'Ancien Régime, fuyant leur dépôt pour aller dans un autre rejoindre des camarades. D'autres s'enfuient après avoir commis un délit. En effet, si quelques prisonniers caressent l'espoir que les autorités ne resteront pas insensibles à la précarité de leur existence, tels ces officiers voire ces sous-officiers qui n'hésitent pas à demander des avances, il en est qui, par désespoir, quittent les chemins de la légalité. Mendicité, larcins, vols avec effraction, de telles actions dégradent leur image auprès de la population locale.

Le dernier chapitre examine, autant que l'état des sources le permet, la question complexe des rapports entre les étrangers et les Français du Puy-de-Dôme. L'auteur montre qu'il existe chez les révolutionnaires du département une volonté de combattre l'image négative que ces captifs peuvent avoir de la Révolution, et un prosélytisme de conversion qui ne porte pas de fruits, les prisonniers persistant à se considérer comme des otages. D'autant que, comme le révèlent des passages éclairants, ils gardent une cohésion de groupe et, par conséquent, qu'ils maintiennent les structures qu'ils ont connues auparavant dans leurs propres sociétés. D'abord, parce que chaque dépôt conserve son caractère «national et que les officiers, encore moins les sous-officiers dont la place est primordiale, sont rarement séparés de leurs hommes. Les hiérarchies antérieures, acceptées par les soldats, persistent, la captivité ne joue pas le rôle niveleur qu'elle aurait pû avoir. Par ailleurs, et de façon contradictoire avec leur pédagogie révolutionnaire, les autorités réagissent parfois pour limiter les contacts des étrangers avec les civils qui auraient pu transformer leur vision des choses. Elles craignent la «réciprocité, par exemple de la part des Piémontais «fanatiques. Des prisonniers sont soupçonnés d'avoir joué avec l'émotion populaire, au moment de la réaction thermidorienne, puis en 1799.

9 La libération met fin à ces contacts, mais pas pour tous. Certains choisissent de devenir citoyens français et -ou- de se marier avec une Française. On trouve à la fin de ce chapitre l'évocation très vivante d'un Autrichien, père d'un chef d'orchestre français qui fit carrière au XIX ${ }^{e}$ siècle. Quant à ceux qui revinrent chez eux, on ne sait s'ils se firent missionnaires des idées nouvelles. Sur ce point, on reste un peu sur sa faim mais l'auteur avoue le silence des sources. 
10 Cet ouvrage précis et dense se termine par deux index, l'un thématique et l'autre des noms de personnes, fort utiles pour le lecteur. 